\title{
The Paradoxes of EMT Theory in Carcinoma Metastasis
}

\author{
Mingyang Li \\ Department of Pathology and Pathophysiology, Fourth Military Medical University, Xi'an, China \\ Email:limingyang1108@sina.com
}

Received 7 June 2014; revised 12 July 2014; accepted 22 August 2014

Copyright (C) 2014 by author and OALib.

This work is licensed under the Creative Commons Attribution International License (CC BY). http://creativecommons.org/licenses/by/4.0/

(c) † Open Access

\begin{abstract}
The epithelial-to-mesenchymal transition (EMT) is a process where epithelial cells with polarity and tight junction change into mesenchymal cells with out polarity and intercellular adhesion to obtain an ability of invasion and metastas is. Interest in the EMT theory has grown exponentially in the last several years and it has become one of the hottest subjects in cancer research. However, there is no convincing evidence of EMT in human tumor and numerous paradoxes do exist about EMT theory from molecular researches to clinical observations. Thus, the so-called EMT in carcinoma metastasis is probably a pseudo concept at present.
\end{abstract}

\section{Keywords}

\author{
Carcinoma Metastasis, Epithelial-to-Mesenchymal Transition (EMT), Paradoxes \\ Subject Areas: Molecular Biology, Pathology
}

\section{Introduction}

Carcinoma is by far the most prevalent form of cancer, with $>90 \%$ of all human malignancies derived from epithelial orig in. As to about $90 \%$ of cancer deaths, cancer metastasis is what ultimately kills the patient. It is extremely important and essential to make clear the mechanis $\mathrm{m}$ of carcinoma metastasis and how to block it. But the whole area of metastas is has been "one of unfathomable complexity" [1]. In recent years, a potential mechanism for metastasis is becoming very popular - the epithelial-to-mesenchymal transition (EMT), which is a normal process during specific stages of embryonic development where epithelial cells transform into mesenchymal cells giving rise to the development of bone, muscle, connective tissue, and blood vessels. EMT is really becoming one of the hottest subjects and there are more and more data coming out for the role of EMT in metastasis. EMT “is one of the things that everybody's doing nowadays, and I can't tell you how many papers I'm getting to review about EMT [1]."

During the progression of carcinoma, advanced tumor cells frequently exhibit a conspicuous downregulation 
of epithelial markers and a loss of intercellular junctions, resulting in a loss of epithelial polarity and reduced intercellular adhesion. The loss of epithelial features is often accompanied by increased cell motility and expression of mesenchymal genes. This process, referred to as epithelial to mesenchymal transition (EMT), can promote hallmark features of carcinoma, including loss of contact inhibition, altered growth control, and enhanced invasiveness [2].

Despite the popularity of EMT theory and masses of proponents, it is a controversial subject and there are numerous paradoxes from molecular researches to clinical observations about EMT theory in carcinoma metastasis. Scientific researchers have accepted certain "paradigm" before starting their work. The target they focus on is not natural at all, but in a box full of conceptions and logics. So what if the so-called paradig $\mathrm{m}$ is fake and erroneous? Therefore, it is very necessary to gain a clear idea of whether the EMT theory is right or wrong in order to let us avoid trouble and make contributions to cancer research.

\section{Pathologists Haven't Seen EMT in Clinical Tumor Samples}

"In $>40$ years experience of reporting on the pathology of many thous ands of tu mor samples, the author has not seen convincing evidence of EMT at any stage of neoplasia, from the conversion of in situ into invasive carcinoma, nor during progression to the most advanced stage IV lesions with systemic metastases [3]." In the review of Tarin D, He illustrated some samples which are thought to be the EMT but have the representative feature of epithelial cells with close scrutiny. Thus, he dismissed EMT as a "fallacy" and called it "an unfortunate misconception resulting from erroneous interpretation of pathologic data" [3].

In spite of finding a number of EMT markers and signaling pathways even genetic elements on the bas is of results from cultured cells and animal models, it is dubious that EMT is true without convincing morphologic evidence in human tumor. Robert Weinberg suggested that this may simply because EMT is so transient-once a metastatic cell has invaded a new tissue, its mesenchymal features melt away. But Tarin D thought it's comparable to one telling you there are invisible aliens sitting in this room, because you haven't used the right tools, you haven't seen them [4]. From the criterion of Carl Popper's philosophy, the statement that EMT is too transient to observe is not falsifiable and the EMT theory in carcinoma metastasis cannot be regarded as a scientific theory.

\section{Some Molecular Markers and Morphologic Features of EMT Aren't Bound up with Carcinoma Metastasis Intimately}

E-cadherin, which is a transmembrane protein localized to the adherens junctions and basolateral plasma membrane, represents the best-characterized molecular marker expressed in epithelial cells. Expression of vimentin is observed in mesodermal cells during specific stages of embryonic development and is associated with a highly invasive cellu lar phenotype [2]. In EMT theory, down-regulation of E-cadherin and up-regulation of vimentin is a common feature in many forms of carcinoma and can often predict invasiveness and metastatic potential. However, in a spontaneous murine breast cancer's research, 67NR cells, which form primary tumors but fail to metastasize, express vimentin but not E-cadherin. 4T1 cells express E-cadherin but are invasive and metastasize to multiple sites [5]. Moreover, focal vimentin expression has been reported variably in about $15 \%$ of invasive breast cancer studies [6]. These researches suggest that E-cadherin and vimentin may not be clear indicators of EMT in breast cancer, the same as salivary neoplasm [7] and gastric carcinoma [8].

By reviewing the definition of EMT at the beginning of this article, we know the outcome of EMT is epithelial cells change into mesenchymal cells obtaining an ability of invasion and metastasis. Therefore, epithelial morphology should not be in invasive and metastatic carcinomas. But there are several reported examples of carcinoma cells within primary and metastatic lesions with well-differentiated epithelial morphology such as prostate cancer [9], mammary ductal carcinoma [10] and salivary neoplasms [7]. It is concluded that the socalled features and markers of EMT correlate poorly with carcinoma metastasis and EMT theory be dispensable to certain cancer metastasis.

\section{Mesenchymal Cells in Certain Tissues Derived from EMT Aren't Motile in Vivo and the Presence of EMT in Carcinoma Cells Correlates Poorly with Cancer Prognosis}

Mesenchymal cell gives the impression of much more relaxed organization, and this suggests flexibility, indi- 
vidualis $\mathrm{m}$, and motile propensities [11]. However, many mesenchymal cells derived from EMT show poor ability to move. Sclerotomal mesenchyme cells are motile in vitro, but these cells have little migratory potential in vivo, as shown by grafting them into neural crest mesenchyme mig ration pathways [12]. Moreover, fibroblast cells are produced by an EMT-like mechanism in fibrotic kidney, and these cells remain local [13]. These researches manifest that not all cells from EMT process can obtain an ability of invasion and metastasis, so the evidence of metastasis of cancer cells which have undergone EMT is insufficient.

Metastasis is the most serious outcome of cancer for about $90 \%$ of cancer deaths coming from distant metastasis to other organs. Following th is point of view, patients whose cancer cells undergo the EMT process should have worse prognosis than those don't. Similarly, the presence of epithelial polarity and high expression levels of E-cadherin in carcinoma cells should contribute to preventing cancer metastasis. But in a research of breast tumor, the presence of epithelial polarity in invasive carcinoma cells failed to prognosticate presence of metastatic lesions and patient survival [14]. Additional investigations have recapitulated these findings and showed that E-cadherin status correlated poorly with disease recurrence, distant metastases, vascular invasion, or other prognostic factors [15]. Taken together, these observations suggest that EMT theory is not required for carcinoma metastasis.

\section{The Concept of EMT Coming from Embryonic Development Isn't Suitable for Tumor Cells with Genetic Instability}

The process of embryonic development consists of highly ordered series of events. Applying the concept of EMT derived from embryonic development to the disorganized series of events during tumor progression isn't reasonable originally. Additionally, EMT markers are unreliable anyway because tumors are genetically unstable. Some inappropriate expressions in genetically unstable cells could be responsible for shifts in single markers of lineage. The gain or loss of one or a few markers is insufficient to conclude that a cell population has implemented whole-scale gene expression reprogramming to abandon one life-style and adopt another which is totally different [3].

"People really want these cells to do an EMT and they push the concept too far" [4]. Many papers report results from cultured cells without confirming that the cells are metastatic in live animals. The EMT theory in carcinoma metastasis, derived from comparisons with embryologic data, is based upon imagined similarities to some microscopic features of human tumors [3]. Doing a scientific research isn't like writing a science fiction, which needs precise data and accurate observations, not excessive imag inations. Therefore, the EMT theory in carcinoma metastasis isn't trustworthy to a certain extent.

\section{Conclusion}

A scientific theory must not only be verified but also be falsified. There is no convincing evidence of EMT in human tumor, but somebody also suggests that EMT is too transient to observe. So the EMT theory in carcinoma metastasis is neither verified nor falsified. Although many molecular biological investigations support for the hypothesis of EMT theory, numerous paradoxes do exist from molecular researches to clinical observations. Thus, we believe that the EMT in carcinoma metastasis is probably a pseudo concept until it is completely proved.

\section{References}

[1] Garber, K. (2008) Epithelial-to-Mesenchymal Transition Is Important to Metastasis, but Questions Remain. Journal of the National Cancer Institute, 100, 232-233,239. http://dx.doi.org/10.1093/jnci/djn032

[2] Christiansen, J.J. and Rajasek aran, A.K. (2006) Reassessing Epithelial to Mesenchy mal Transition as a Prerequisite for Carcinoma Invasion and Metastasis. Cancer Research, 66, 8319-8326. http://dx.doi.org/10.1158/0008-5472.CAN-06-0410

[3] Tarin, D., Thompson, E.W. and Newgreen, D.F. (2005) The Fallacy of Epithelial Mesenchymal Transition in Neoplasia. Cancer Research, 65, 5996-6000. http://dx.doi.org/10.1158/0008-5472.CAN-05-0699

[4] Ledford, H. (2011) Cancer Theory Faces Doubts. Nature, 472, 273. http://dx.doi.org/10.1038/472273a

[5] Lou, Y., Preobrazhenska, O., auf dem Keller, U., Sutcliffe, M., Barclay, L., McDonald, P.C., Roskelley, C., Overall, C.M. and Dedhar, S. (2008) Epithelial-Mesenchymal Transition (EMT) Is Not Sufficient for Spontaneous Murine Breast Cancer Metastasis. Developmental Dynamics, 237, 2755-2768. http://dx.doi.org/10.1002/dvdy.21658 
[6] Gilles, C. and Thompson, E.W. (1996) The Epithelial to Mesenchymal Transition and Metastatic Progression in Carcinoma. The Breast Journal, 2, 83-96. http://dx.doi.org/10.1111/j.1524-4741.1996.tb00076.x

[7] Economopoulou, P., Hanby, A. and Odell, E.W. (2000) Expression of E-Cadherin, Cellu lar Differentiation and Polarity in Epithelial Salivary Neoplasms. Oral Oncology, 36, 515-518. http://dx.doi.org/10.1016/S1368-8375(00)00043-9

[8] Gabbert, H.E., Mueller, W., Schneiders, A., Meier, S., Moll, R., Birchmeier, W. and Hommel, G. (1996) Prognostic Value of E-Cadherin Expression in 413 Gastric Carcinomas. International Journal of Cancer, 69, 184-189. http://dx.doi.org/10.1002/(SICI)1097-0215(19960621)69:3<184::AID-IJC6>3.0.CO;2-W

[9] Christiansen, J.J., Rajasekaran, S.A., Inge, L., Cheng, L., Anilkumar, G., Bander, N.H. and Rajasekaran, A.K. (2005) N-Glycosylation and Microtubule Integrity Are Involved in Apical Targeting of Prostate-Specific Membrane Antigen: Implications for Immunotherapy. Molecular Cancer Therapeutics, 4, 704-714. http://dx.doi.org/10.1158/1535-7163.MCT-04-0171

[10] Ng, W.K. (2002) Fine-Needle Aspiration Cytology Findings of an Uncommon Micropapillary Variant of Pure Mucinous Carcinoma of the Breast: Review of Patients over an 8-Year Period. Cancer, 96, 280-288. http://dx.doi.org/10.1002/cncr.10747

[11] Thompson, E.W., Newgreen, D.F. and Tarin, D. (2005) Carcinoma Invasion and Metastasis: A Role for EpithelialMesenchy mal Transition? Cancer Research, 65, 991-995. http://dx.doi.org/10.1158/0008-5472.CAN-05-0616

[12] Erickson, C.A., Tosney, K.W. and Weston, J.A. (1980) Analy sis of Migratory Behavior of Neural Crest and Fibroblastic Cells in Embryonic Tissues. Developmental Biology, 77, $142-156$. http://dx.doi.org/10.1016/0012-1606(80)90462-5

[13] Kalluri, R. and Neilson, E.G. (2003) Epithelial-Mesenchymal Transition and Its Implications for Fibrosis. The Journal of Clinical Investigation, 112, 1776-1784. http://dx.doi.org/10.1172/JCI200320530

[14] Tan, D.S., Potts, H.W., Leong, A.C., Gillett, C.E., Skilton, D., Harris, W.H., Liebmann, R.D. and Hanby, A.M. (1999) The Biological and Prognostic Significance of Cell Polarity and E-Cadherin in Grade I Infiltrating Ductal Carcinoma of the Breast. The Journal of Pathology, 189, 20-27. http://dx.doi.org/10.1002/(SICI)1096-9896(199909)189:1<20::AID-PATH394>3.0.CO;2-2

[15] Parker, C., Rampaul, R.S., Pinder, S.E., Bell, J.A., Wencyk, P.M., Blamey, R.W., Nicholson, R.I. and Robertson, J.F. (2001) E-Cadherin as a Prognostic Indicator in Primary Breast Cancer. British Journal of Cancer, 85, 1958-1963. http://dx.doi.org/10.1054/bjoc.2001.2178 